\title{
Defect Detection and Classification using a SQUID based multiple Frequency Eddy Current NDE System
}

\author{
M. v. Kreutzbruck, K. Allweins, T. Rühl, M. Mück and C. Heiden \\ H.-J. Krause, R. Hohmann
}

\begin{abstract}
The probability of detection (POD) of hidden fatigue defects in riveted multilayer joints, e.g. aireraft fuselage, can be improved by using sophisticated eddy-current systems which provide more information than conventional NDE equipment. In order to collect this information, sensor arrays or multi-frequency excitation schemes can be used. We have performed simulations and measurements with an eddy current NDE system based on a SQUID magnetometer. To distinguish between signals caused by material defects and those caused by structures in the sample, such as bolts or rivets, a high signalto-noise ratio is required. Our system provides a large analog dynamic range of more than $140 \mathrm{~dB} / \sqrt{\mathrm{Hz}}$ in unshielded environment, a digital dynamics of the ADC of more than 25 bit $(>150 \mathrm{~dB}$ ) and multiple frequency excitation. A large number of stacked aluminum samples resembling aircraft fuselage were measured, containing titanium rivets and hidden defects in different depths in order to obtain sufficient statistical information for classification of the defect geometry. We report on flaw reconstruction using adapted feature extraction and neural network techniques.
\end{abstract}

\section{Index Terms- Feature Extraction, Nondestructive Testing,} SQUIDs.

\section{INTRODUCTION}

I

ncreasing requirements in quality control demand the reliable detection of very small or deep lying defects hidden in conductive samples, such as aircraft parts. The development of new analysis techniques, based on both software algorithms and adapted hardware components, is thus becoming increasingly important for crack detection based on electromagnetic testing. The question whether sophisticated hardware equipment of eddy current (EC) systems with higher field sensitivity and higher dynamic range actually meets these requirements is discussed with respect to special EC applications.

SQUID EC NDE is in competition with numerous other NDE techniques, such as X-ray and ultrasound. Especially Xray investigations including inverse modelling usually allow for a reliable estimation of the defect geometry. However, in the case of eddy current testing the electromagnetic inverse problem has been entirely solved only for $2 \mathrm{D}$ problems or a simple 3D-geometry of the sample [1], but not when Manuscript received September 18, 2000. This work was supported by the German BMBF under Grant No. 13N7427/9.

This work is dedicated to the memory of Christoph Heiden and James E. Zimmerman.

M. v. Kreutzbruck, K. Allweins, T. Rühl, M. Mück are with the Institut für Angewandte Physik, J.-L.-Universität Giessen, 35392 Giessen, Germany. H.-J. Krause and R. Hohmann are with the Institut für Schicht- und Ionentechnik (ISI), Forschungszentrum Jülich, D 52425 Jülich, Germany. measuring complicated 3D- samples using complicated excitation schemes. Besides numerical modelling approaches such as Finite-Element-Method (FEM) and the VolumeIntegral-Method (VIM) many efforts have been undertaken to solve the inverse problem using neural networks for a classification of the defect geometry [2],[3]. For this purpose as much as possible information is required. In order to obtain more specific data about the sample under test, sensor arrays, sensors with different alignments measuring different field components or several excitation frequencies can be used.

In addition to the amount of information also the quality of the measured field distribution plays an important role for a successful feature extraction and classification of material defects. In standard EC testing, the field data are usually recorded in a 12 to 16 bit format, corresponding to an analog dynamic range of 65 to $85 \mathrm{~dB}$. But when measuring samples with complicated geometrical structures and consisting of different materials, defect signals are superimposed by, for example, edge effects, which often are several magnitudes larger than the field variation caused by the defect. Furthermore, in standard EC signal processing algorithms, such as subtraction methods and phase rotation, the defect signal must be separated from an artificial background, which requires sometimes, in the case of small defects, a dynamic range of more than 16 bit to classify the defect signal.

A SQUID proves to be a promising sensor, because it provides by combination of high field sensitivity and an outstanding high dynamic range for the detection of very small material defects [4]-[9].

\section{DETERMINATION OF OPTIMUM EXCITATION FREQUENCY}

In order to obtain a maximum eddy current density at the defect, both inducer and excitation frequency must be chosen correctly. To obtain the optimum excitation frequency for a given defect depth, we calculate the induced current density of an incident electromagnetic plane wave, which can be written as: $j(z)=j_{0} e^{-z / \delta}$, where

$$
\delta=\frac{1}{\alpha}=\sqrt{\frac{2}{\omega \cdot \sigma \cdot \mu_{0} \cdot \mu}} .
$$

$\mathrm{Z}$ is the defect depth and $\delta$ is the skin depth ( $\omega$ : frequency, $\sigma$ : conductivity, $\mu$ : permeability). The skin depth denotes the material depth in which the amplitude has decreased to 1/e of its value at the surface and the phase is rotated by $\pi$. However, when using a small coil rather than a planar incident wave to excite eddy currents, both the surface current 
density $\mathrm{j}_{0}$ and the real penetration depth $\delta=1 / \alpha$ will be different from the values calculated using (1). To account for this effect, we can determine corrected values $j_{\mathrm{c} 0}$ and $\alpha_{\mathfrak{c}}$,

$$
j_{c}=j_{c 0} e^{-\alpha_{c} z} \text {, }
$$

which have been calculated for different kinds of coil geometries in [10].

Four components contribute to the variation of the eddy current field $\Delta \mathrm{B}_{2}$ caused by a defect: (i) the current density at the defect, described by (2), (ii) the change in current flow and thus local current density caused by the defect, which we describe by a factor $\lambda$, (iii) the attenuation of a magnetic field measured at the surface of the aluminium sample but generated by a defect current flowing in a depth $z$, which is proportional to $\exp \left[-\alpha_{\text {defect }} \cdot z\right]$ and (iv) the spatial decay of the quadrupole field of the defect, if the distance between defect and sensor is larger than the defect size. This is proportional to a function $\mathrm{f}(\ell / \mathrm{z})$, yielding a $\ell / \mathrm{z}$ dependence in the case when the length $\ell$ of the crack is smaller than its depth $z$ in the material, and unity when $\ell>\mathrm{z}$. This can be summarized by the following expression:

$$
\Delta B_{z}=j_{c 0}(\omega) \cdot e^{-\alpha_{c}(\omega, z) \cdot z} \cdot \lambda \cdot e^{-\alpha_{\text {crack }}(\omega, z) \cdot z} \cdot f\left(\frac{\rho}{z}\right)
$$

We note that due to the induction law the current density at the surface of the sample $\mathrm{j}_{0}$ is also frequency dependent. For too high frequencies the current density at the surface scaling with $\sqrt{\mathrm{Hz}}$ - is sufficient high but the penetration depth decreases, thus leading to an exponential reduced response from deep lying defects. Otherwise for too low excitation frequencies the current density at the surface is too small which results despite a larger penetration depth in a lower field variation at the location of the crack. Equation (3) can be used to calculate the optimum excitation frequency (see also Fig.1).

As an example Fig. 1 shows the optimum excitation frequency for a defect located in a depth of $14 \mathrm{~mm}$ in case of an aluminium specimen, having an electrical conductivity of $22 \mathrm{MS} / \mathrm{m}$. One can recognize a maximum response field when using a frequency of about $185 \mathrm{~Hz}$. Here a double-D coil with $30 \mathrm{~mm}$ in dia. was assumed for the calcualtion of the magnetic response field.

For real samples, e.g., multi-layer samples containing titanium rivets, in some cases the excitation frequency should not be chosen to give the maximum eddy current density at the defect, but to provide an easy distinction between a defect

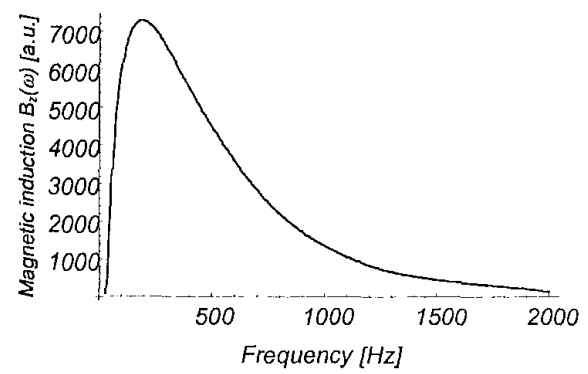

Fig. 1. Magnetic field amplitude of the response when measuring a defect located in a depth of $14 \mathrm{~mm}$ with a double-D coil with $30 \mathrm{~mm}$ in diameter.

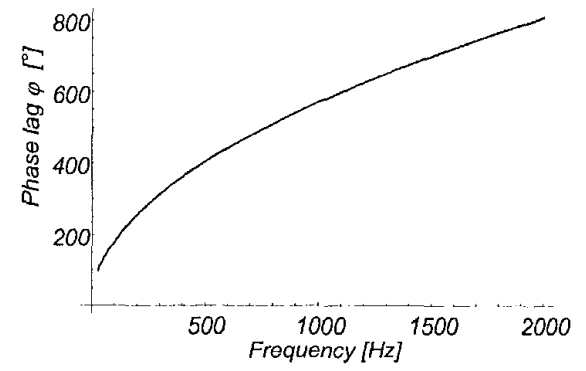

Fig. 2. Phase lag $\varphi$ between the field response of a defect located in a depth of $14 \mathrm{~mm}$ and the field response caused by the upper part of the rivet (calculated by 3D-FEM).

and a rivet. For this purpose one can make use of the depth dependent phase shift between the magnetic fields generated by defects and rivets in different depths. Fig. 2 shows the phase shift between the magnetic field of a defect in a depth of $14 \mathrm{~mm}$ and the field of the upper part of a titanium rivet. By changing the excitation frequency, a wide range of phase shifts can be covered.

In order to suppress the disturbing signal of the rivet, the excitation frequency can be chosen such that there is a phase shift between defect and rivet signal of $(2 n-1) \cdot \pi / 2$. In this case, the in-phase output of the lock-in amplifier contains only the defect signal, whereas the quadrature output contains only the rivet signal, which is needed in some cases for further data processing.

When testing multilayer aircraft parts, defects usually appear in a certain layer and in a constant depth due to characteristic stress loading, leading to a quite constant phase lag between the defect signal and the response of the rivet. Therefore at least one frequency $f_{n}$ can be found, where the phase lag is $90^{\circ}, 270^{\circ}$ or $450^{\circ}$. Fig. 3 shows the sine function of the phase lag,

$$
g(\varphi, \omega)=\sin [\varphi(\omega)],
$$

indicating these optimum excitation frequencies at the positions of the extrema values.

In order to achieve an optimum signal to noise ratio of the defect response, both (3) and (4) have to be taken into consideration. Multiplication of (3) and (4) gives the amplitude of the defect response in one channel of the lock-in amplifier for a certain phase shift between excitation field and lock-in reference. As an example Fig. 4 shows that in the case

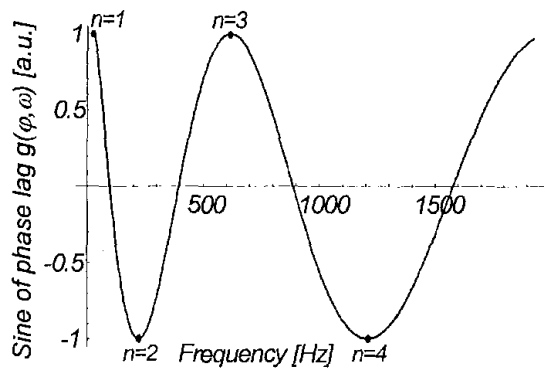

Iig. 3. Sine function of the phase lag between defect and rivet signal, used for minimizing the signal of the rivet by phase rotation. At positions of the extrema values the phase angle between crack signal and rivet signal is (2n-1) $90^{\circ}$. 


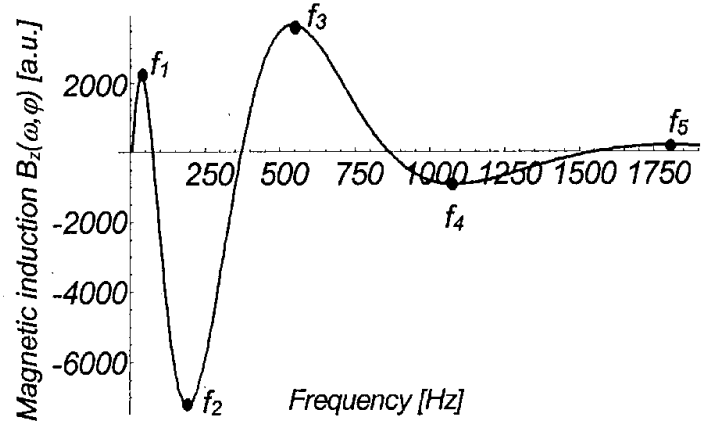

Fig. 4. Optimum excitation frequency for maximum defect signal, while simultaneously minimizing the signal of a rivet by phase rotation.

of a defect hidden in an aluminium sample in a depth of 14 $\mathrm{mm}$, several excitation frequencies are possible, which give a phase shift of $(2 n-1) \cdot \pi / 2: f_{1}: 35 \mathrm{~Hz}(31 \%$ signal strength with respect to (3)), $\mathrm{f}_{2}: 195 \mathrm{~Hz}(98 \%), \mathrm{f}_{3}: 540 \mathrm{~Hz}(51 \%), \mathrm{f}_{4}: 1085$ $\mathrm{Hz}(11 \%), \mathrm{f}_{5}: 1800 \mathrm{~Hz}(2.7 \%)$.

\section{MEASUREMENT CONFIGURATION}

In our measurements we used three excitation frequencies. Two of them are used to provide depth selective information about the defect and the third frequency is used to localize the position of the rivet, which is required for better distinction between rivet and defect signals.

Figure 5 shows the principle block diagram of the measurement configuration. The excitation unit generates signals of frequency $f_{1}$ to $f_{3}$, each of which is fed to an individual coil to excite eddy currents in the sample.

As excitation coils we usually use either a gradiometric double-D coil or a circular coil $\left(f_{1}\right)$ and two wires $\left(f_{2}, f_{3}\right)$. The wires are perpendicular to each other and their crossover is located above the center of the double-D coil. The SQUID is located right above the crossover of the two wires, which are attached to a small $x / y$-stage in between the cryostat and the sample and are positioned such that the excitation field at the SQUID is as low as possible.

If there is a flaw in the sample, the current distribution and thus the field distribution above the flaw changes. The corresponding inhomogeneity in the magnetic field is detected by one single SQUID. Three lock-in amplifiers measure amplitude and phase of the response signal from the sample at the three excitation frequencies. In order to prevent each lock-in amplifier from being influenced by the two other excitation frequencies the bandwidth of the lock-in amplifiers was chosen to be smaller than $30 \mathrm{~Hz}$.

The demodulated field data are read into a personal computer, using 16 bit analog-to-digital converters. In addition the two output channels of the first lock-in amplifier $f_{1}$ can be measured with a high resolution multimeter, covering a digital dynamic range of up to 28 bit (170 dB). A maximum measuring range of \pm 10 Volts and a noise amplitude of the last 2 bits - corresponding to approximately $1800 \mathrm{nV}$ - was obtained for a bandwidth of about $1 \mathrm{~Hz}$, leading to a maximum dynamic range of $141 \mathrm{~dB} / \sqrt{\mathrm{Hz}}$.

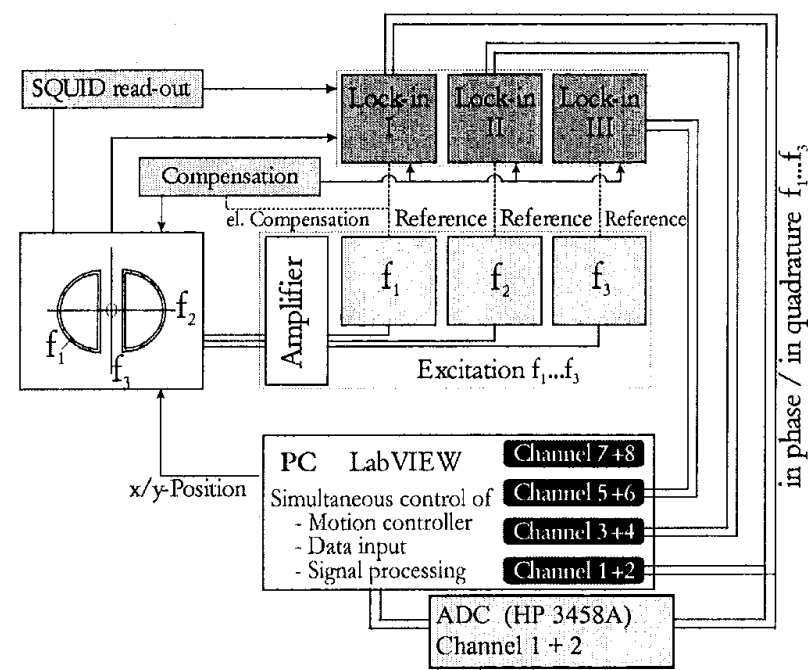

Fig.5. Schematic diagram of the three-frequency measurement configuration.

The sampling rate of this multimeter depends on the required field resolution. When scanning with a resolution of $21 \mathrm{Bit}(126 \mathrm{~dB})$ and a velocity of $30 \mathrm{~mm} / \mathrm{s}$, the sampling rate is limited to $600 \mathrm{~Hz}$, leading to a spacing between the recorded data points of $50 \mu \mathrm{m}$. The measured field data are synchronized with the position of the $\mathrm{x} / \mathrm{y}$-stage. All parameters such as scanning range, velocity, digital dynamic range, bandwith etc, can be controlled by a PC. The system includes features such as extraction of line scans, phase rotation and standard filters.

In our measurements, we used both HTS-rf-washer SQUID magnetometers [11] biased at $900 \mathrm{MHz}$ and microwave rfSQUIDs biased at $3 \mathrm{GHz}$ [12]. The field sensitivity of the washer SQUID was about $700 \mathrm{fT} / \mathrm{JHz}$ at signal frequencies above $10 \mathrm{~Hz}$, and the field to flux transfer coefficient was about $15 \mathrm{nT} / \Phi_{0}$. The SQUID was cooled using a JouleThomson cooler. The spacing between SQUID and sample was about $5.5 \mathrm{~mm}$. The size of the SQUID washer was 2.5 $\mathrm{mm}$, leading to only a negligible reduction in the spatial resolution of the system. Further information of the cooling and SQUID integration are described elsewhere [13].

\section{MEASUREMENTS}

\section{A. Measurements of riveted multi layer samples}

Using the measurement configuration described above, we measured a number of multi-layer samples of different geometry. A test sample provided by EADS Airbus, which should resemble thick parts of the Airbus A3XX aircraft wing and fuselage, is shown in Fig. 6 (bottom right). Several layers of aluminium sheet are bolted together with a $8 \mathrm{~mm}$ dia. titanium rivet. The thickness of the individual layers could be varied between 2 and $10 \mathrm{~mm}$. The second or third layer contains an exchangeable sheet, in which defects with different lengths $\ell$ have been introduced adjacent to the rivet. The conductivity of the aluminium was about $22 \mathrm{MS} / \mathrm{m}$, whereas the conductivity of the rivet, made from titanium alloy, is more than one order of magnitude smaller, leading to strong current distortions in the vicinity of the rivet. 

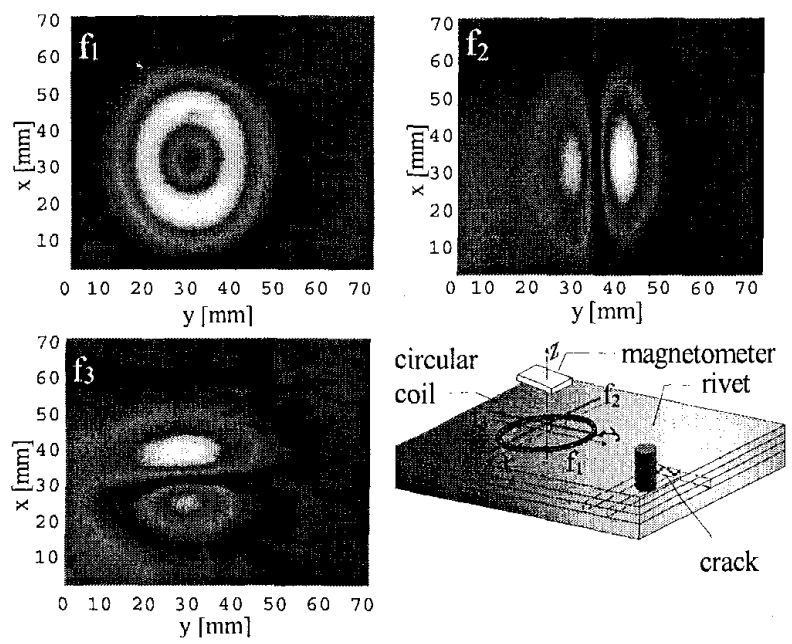

Fig. 6. Field distribution above a riveted sample for three frequencies $\mathrm{f}_{1}$ :circular coil excitation at $410 \mathrm{~Hz}, \mathrm{f}_{2}$ : wire excitation at $1.28 \mathrm{kHz}, \mathrm{f}_{3}$ : wire excitation at $2.55 \mathrm{kHz}$, defect length: $5 \mathrm{~mm}$, heigth: $5 \mathrm{~mm}$, depth: $5 \mathrm{~mm}$ ). Raw data with arbitrary phase rotation, measured with a $3 \mathrm{GHz}$ if SQUID

Fig. 6 shows the measured field distribution above the sample for three different excitation frequencies. No adapted phase rotation was performed and only the field variation caused by the rivet can be recognized. The defect signal is much smaller than the superimposed rivet signal and thus cannot be seen in the raw data. For this measurement a microwave-rf SQUID was used in combination with a circular excitation coil, leading to a somewhat simpler signal compared to a double-D coil. The response fields at the frequencies $f_{1}$ and $f_{2}$ are used for feature extraction, whereas the response field at $f_{3}$ is only used for the determination of the position of the rivet. This is required for some signal processing algorithms.

We note that the estimation of optimum excitation frequencies due to the orthogonality of the in-phase and the quadrature channel also allows for a suppression of the defect signal and to maximize the rivet signal by choosing a certain rotation angle $\varphi$. In conjunction with higher excitation frequencies $(n \geq 5)$ this makes a reliable estimation of the position of the rivet possible.

\section{B. Signal processing}

In order to separate the defect signal from the background, several steps in signal processing have to be performed. As an example in Fig. 7 we show a line scan across the rivet and the resulting field after several of these signal processing steps. First, the Lift-Off effect (change of response field due to varied spacing between SQUID and sample) has to be eliminated by subtracting a linear function (see Fig. 7, line 2). The second step contains a phase rotation to minimize the rivet signal and enhance the defect signal (see Fig. 7, line 3). Nevertheless, the defect signal is still superimposed by a signal from parts of the rivet which are in the same depth as the defect. Therefore, as a third step for further suppression of the rivet signal, a "reference" rivet signal is subtracted. The residual signal then only contains field variation which are caused by the defect, having two characteristic local minima,

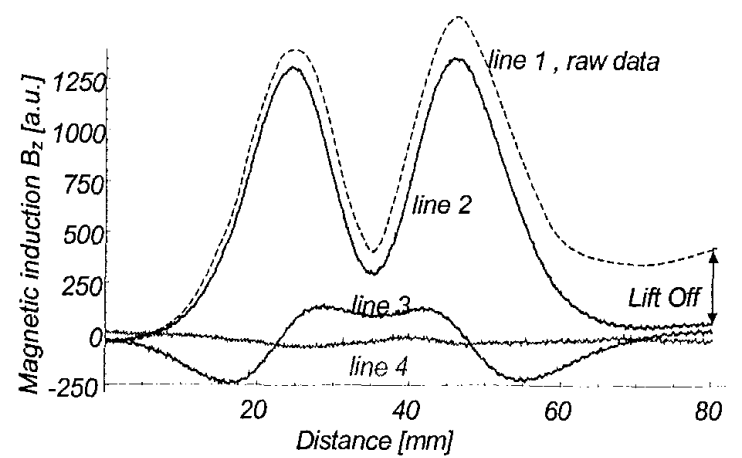

Fig. 7. Line scan across a riveted multilayer sample using $180 \mathrm{~Hz}$ excitation frequency. A $5 \mathrm{~mm}$ long crack was located in a depth of $10 \mathrm{~mm}$. Line 1: raw data containing both rivet and defect signal. Line 2 : suppression of the lift-off effect by subtracting a linear function. Line 3 : suppression of the rivet signal by rotating the phase. Line 4 : subtracting a "reference" rivet signal to minimize the residual rivet signal.

due to the excitation with a circular coil (see Fig. 7, line 4). Each of these signal processing operations yield a reduction of rivet signal amplitude of up to one order of magnitude. Especially when measuring defects in larger depths, the crack signal could be smaller by 3 orders of magnitude compared to the rivet signal.

\section{Measuring with high digital resolution}

We note that the above described algorithm unfortunately results in a reduced SNR of the defect signal, especially if the dynamic range of the ADC used to collect the data is small $(<16$ bit). This is shown in Fig. 8, where we plot defect signals of a $5 \mathrm{~mm}$ (top) and a $10 \mathrm{~mm}$ (bottom) long crack in a depth of $10 \mathrm{~mm}$. When using an ADC resolution of only 14 bit (Fig. 8, left) the SNR, after the defect signal enhancement algorithm was applied, was low. Data collected with a 21 bit ADC lead to a much higher SNR (Fig. 8, right). High SNR plays an important role for further classification algorithms, in which features must be determined by inspecting the defect signal. Therefore, in the case of too low digital resolution, this quantization noise inevitably gives rise to a diminished Probability of Detection (POD).
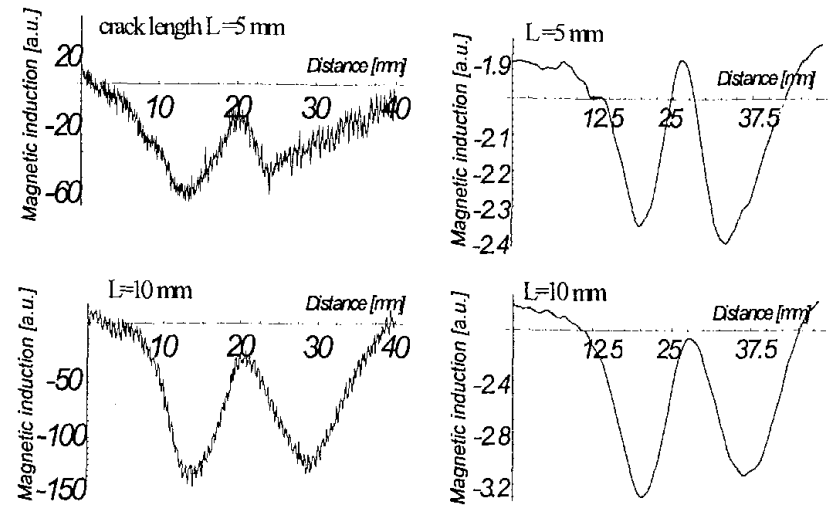

Fig. 8. EC measurement of a riveted multi layer sample, using $180 \mathrm{~Hz}$ excitation frequency. A $5 \mathrm{~mm}, 10 \mathrm{~mm}$ long crack was located in a depth of 10 $\mathrm{mm}$. Suppression of the rivet signal by rotating the phase and subtracting a "reference" rivet signal to enhance the defect response. Left: 14 bit ADC resolution, right: 21 bit ADCresolution was used. 


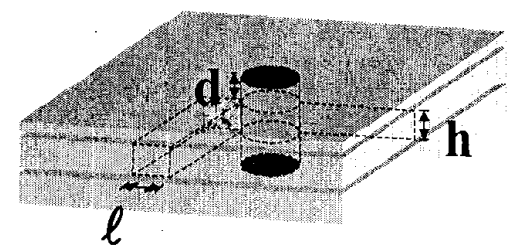

Fig. 9. Sketch of a riveted multi-layer sample, with defect adjacent to rivet.

\section{FEATURE EXTRACTION AND DEFECT CLASSIFICATION}

Modern software algorithms such as neural network techniques, allow for the classification of the defect geometry by extracting features, such as amplitude, width, slope, position of the maxima and the integral function from the response signal [14]. However, the reliable determination of a feature vector from the measured field distribution requires a sufficiently high signal-to-noise ratio. This is especially important when measuring very small and deep lying defects located in close proximity to bolts, rivets and edges. Besides high signal-to-noise ratio we focused to obtain enough statistical information for a defect classification based on neural network techniques. A number of measurements were carried out on multi-layer samples containing defects with different lengths $\ell(0,2,5,10,15 \mathrm{~mm})$. At the beginning we measured defects with a height $\mathrm{h}=2 \mathrm{~mm}$ and which were covered by an aluminium sheet with a thickness of $d=5 \mathrm{~mm}$ (Fig. 9).

A sample with five defects of different length was measured 30 times, leading to 150 response signals in total. For these measurements a double-D coil for excitation in combination with an rf- SQUID with washer geometry was used, leading to a somewhat more complicated defect signal (Fig. 10). The optimum excitation frequency $\mathrm{f}_{1}$ was calculated to be $307 \mathrm{~Hz}$. The $f_{3}$ excitation coil current (wire aligned parallel to the defect) was operated at a frequency of $510 \mathrm{~Hz}$.

Additionally a second measurement was carried out, inspecting 150 defects, each of which had a height of $\mathrm{h}=10 \mathrm{~mm}$ and were covered by a sheet with a thickness of $\mathrm{d}=10 \mathrm{~mm}$. Here the optimum excitation frequency $\mathrm{f}_{1}$ was calculated to be $90 \mathrm{~Hz}$. The $\mathrm{f}_{3}$ excitation current had a frequency of $400 \mathrm{~Hz}$.

In principle for defect detection only a single line scan across the center of rivet is necessary. However, in order to find the center of the rivet it is convenient to first perform a 2D-scan. For the determination of reliable features the line scan right above the center of the rivet was then extracted from the 2D-field distribution. In Fig. 10 we show an example for several defect signals at $f_{1}$ using a double-D excitation coil. In addition we also show the residual signal of a rivet without defect obtained after phase rotation (solid line). The presence of a defect increases $\left|\mathrm{B}_{\mathrm{z}}\right|$ at the opposite side of the rivet and decreases $\left|B_{z}\right|$ just above the defect and also broadens the rivet signal (Fig, 10).

More than 60 mathematical features were extracted from each response signal and each frequency $f_{n}$, such as amplitudes of maxima and minima and their ratio, distance between the maxima and minima, total signal width, integral function and maximum values of the first and second derivatives.

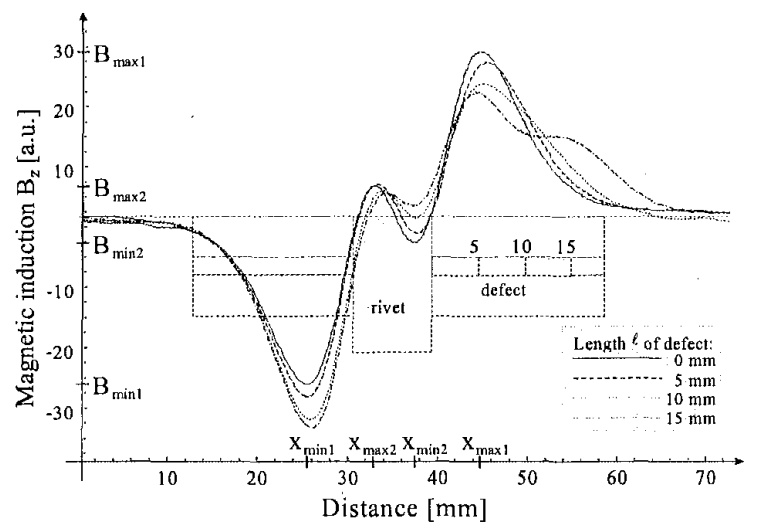

Fig. 10. Magnetic field response of $f_{1}$ after phase rotation for different defect lengths. The defects were located in a depth of $d=5 \mathrm{~mm}$, having a height of $\mathrm{h}=2 \mathrm{~mm}$.

All features $\lambda_{i}$ were normalized to maximum the value of $\lambda_{i}$ obtained from the 150 different data sets and combined into one feature vector $\vec{x}=\left\{\lambda_{1}, \lambda_{2} \ldots\right\}$. Feature vectors of defects with known properties are used to compose a reference vector database. Newly measured data are transformed into a feature vector, which can then be compared to this database in terms of mathematical proximity. The result is given by a function approximator as a weighted superposition of the nearest reference vectors' function values.

As an example Fig. 11 shows the variation of the feature amplitude in dependence on the crack length. Some features can be used to distinguish between rivets without defects

a)

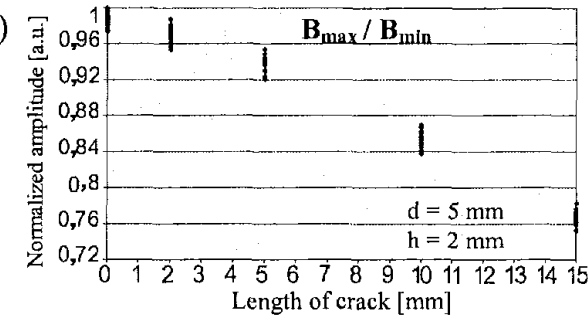

b)

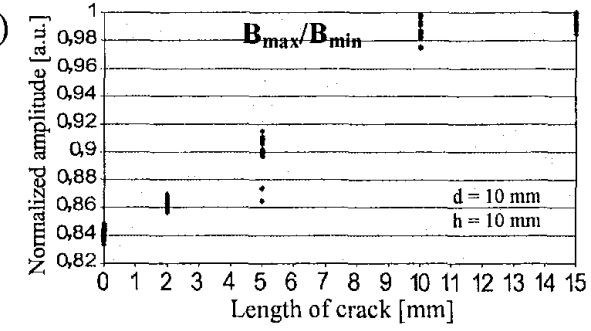

c)

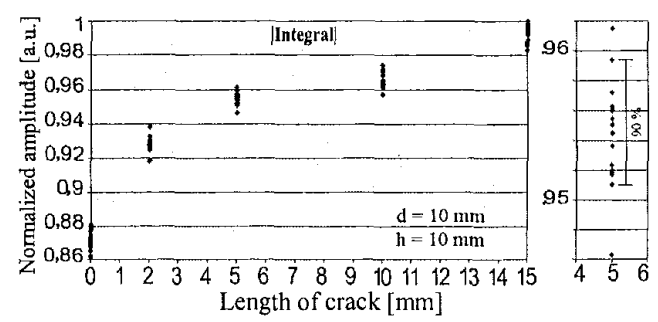

Fig. 11. Examples for normalized feature amplitude at $f_{2}$ for different crack lengths: $\left.a): B_{\max } / B_{\min }(d=5 \mathrm{~mm}, h=2 \mathrm{~mm}), b\right): B_{\max } / B_{\min }(d=10 \mathrm{~mm}, \mathrm{~h}=$ $10 \mathrm{~mm}), \mathrm{c})$ : Integral of absolute values $(\mathrm{d}=10 \mathrm{~mm}, \mathrm{~h}=10 \mathrm{~mm})$. 
and those having defects with lengths larger than $2 \mathrm{~mm}$ (Fig. $11 \mathrm{~b}, \mathrm{c}$ ), whereas other features provide a reliable classification only for defects with lengths larger than $5 \mathrm{~mm}$ (Fig. 11a).

Function approximation of $\mathrm{f}(\mathrm{x})$ - e.g. the defect length $\ell$, depth $\mathrm{d}$ or height $\mathrm{h}$ - is performed by General Regression Networks (standard neural network technique, [15]) utilizing radial bases functions to superimpose unknown function values by the known ones of the nearest reference vectors:

$$
f(\bar{x})=\frac{\sum_{i=1}^{N} f\left(\vec{x}_{i}\right) \cdot w\left(\sigma,\left\|\bar{x}-\vec{x}_{i}\right\|\right)}{\sum_{i=1}^{N} w\left(\sigma,\left\|\vec{x}-\vec{x}_{i}\right\|\right)},
$$

where $\vec{x}$ denotes the unknown feature vector while $\vec{x}_{i}$ represents the reference vectors obtained from the approximator's codebook database. The size of the neighborhood, i.e. the distance dependent influence of reference vectors can be controlled by the neighborhood parameter $\sigma$. A quadratic declining exponential function can be used as weight function $w$. City block metric should be preferred rather than euklidean metric since it is more robust against outliers.

When using conventional eddy current technique, defects hidden in riveted multilayer samples can be found with a probability of $90 \%$, if their length $\ell$ is twice large as their depth $\mathrm{d}$ inside the sample $(\ell / \mathrm{d} \geq 2)$. When using a SQUID sensor with a field sensitivity of better than $\approx 2 \mathrm{pT} / \mathrm{Hz}$, a spatial resolution of better than $\approx 6 \mathrm{~mm}$, multiple frequency excitation and feature extraction, defects with a length of only $2 \mathrm{~mm}$ located in a depth of $\mathrm{d}=10 \mathrm{~mm}$ could be distinguished from defectless rivets in all 150 measurements. Thus our SQUID NDE system was able to detect defects with a size which is one order of magnitude smaller $(\ell / \mathrm{d}=0.2)$ than can be detected with a conventional eddy current system. In case of defects located in a depth of $10 \mathrm{~mm}$, only 7 features were used for a reliable classification of the defect length. We therefore suppose, that the reliable classification of defect depths and defect volume as well as the detection of even smaller defects might be possible.

\section{SUMmary}

The reliable detection of small material defects in riveted multi-layer aircraft samples is difficult, due to the superposition of the small defect response by a large background caused by rivets, bolts and edges of the sample. We could show that the use of a HTS rf SQUID system with three different excitation frequencies and large dynamic range improves the probability of detection (POD) for small defects substantially compared to conventional NDE systems A large number of eddy current measurements were carried out on riveted multi-layer samples resembling real aircraft structures and features were extracted from the measured field distribution to find the lengths of material defects. Optimum system parameters such as different excitation frequencies were calculated and signal processing was used to separate defect signal from high background. Using our HTS rf SQUID system in combination with neural network techniques the determination of the defect lengths hidden in aluminium samples could reliably be achieved.

\section{ACKNOWLEDGMENT}

This work is dedicated to the memory of Christoph Heiden, who initiated the German SQUID NDE Consortium, consisting of EADS Airbus, Lufthansa Technik AG, Rohman GmbH, FZ Jülich, ILK Dresden and JLU Giessen, and to the memory of James E. Zimmerman without whose work on rfSQUIDs this paper would not have been possible.

We thank M. Junger, W. Becker and W.B. Klemmt for the support of aircraft samples and we are grateful to $\mathrm{V}$. Vengrinowich of IAP (Minsk) for many valuable discussions.

\section{REFERENCES}

[1] N.G. Sepulveda, D.J. Staton, and J.P. Wikswo, Jr., "A mathematical analysis of the magnetic field produced by flaws in two-dimensional current-carrying conductors", J. Nondestr. Eval, 11(2): 89-101(1992).

[2] J.P. Wikswo, Jr., "The magnetic inverse problem", in NATO ASI Series, SQUID Sensors: Fundamentals, Fabrication and Applications, $\mathrm{H}$. Weinstock, Fd., Berlin: Springer-Verlag, 1996.

[3] M. Negley, M.R. Govindaraju and D. C. Jiles,"Neural Network Prediction of Creep Damage Based on Magnetic Properties in Power Plant Piping“, Review of Progress in QNDE, Vol 13, S.1817-1824, 1994.

[4] H. Weinstock and M. Nisenoff, "Defect detection with a SQUID magnetometer", Review of Progress in QNDE, 6: 669-704 (1986).

[5] H. Weinstock, R.B. Mignogna, R.S. Schechter, and K.E. Simmonds, "An improved system for the nondestructive evaluation of steel", in Superconducting Devices and Their Applications, H. Koch, H. Lubbig, Eds., Berlin: Springer-Verlag, pp.572-575, 1992.

[6] Y.P. Ma and f.P. Wikswo Jr., "SQUID eddy current techniques for detection of second layer flaws", Review of Progress in $Q N D E, 13: 303-$ 309 (1994).

[7] T.J. Shaw, K. Schlenga, R. McDermott, John Clarke, J.W. Chan, S.-H. Kang and J.W. Morris, "High-Te SQUID Microscope Study of the Effects of Microstructure and Deformation on the Remanent Magnetizition of Stcel", IEEE Trans. Appl. Supercond., vol.9,No. 2 (1999), pp. 4107.

[8] S. Cochran, G.B. Donaldson, C. Carr, D. McA. McKirdy, M.E. Walker, U. Klein, A. McNab, and B. Kuznik, "Recent progress in SQUIDs as sensors for electromagnetic NDE",in IOS Press, Amsterdam, 1995, 53.

[9] F. Schmidl, S. Wunderlich,L. Dörrer, H. Specht, S. Linzen, H. Schneidewind and P. Seidel, "High Tc dc-SQUID system for nondestructive evaluation", IEEE Trans. Appl. Supercond., 7, 2756, (1997).

[10]M.v. Kreutzbruck, M. Mück and C. Heiden, "Simulations about Eddy Current Distributions and Crack Detection Algorithms for a SQUID based NDE System", Proceedings of $7^{\text {th }}$ ECNDT 98, Vol 3, 2513 (1998).

[11]Y. Zhang, M. Mück, K. Hermann, J. Schubert, W. Zander, A.I. Braginski and C. Heiden," Sensitive rf- SQUIDs and magnetometer operating at 77 K“, IEEE Trans.Appl.Supercond. vol.3,(1993), pp. 2465.

[12] Y.Zhang, M.Mück, K.Herrmann, J.Schubert, W.Zander, A.I.Braginski and C.Heiden, Appl.Phys. Lett. 60, pp.2303-2305 (1992).

[13] R. Hohmann, M. Maus, D. Lombarski, M. Grüncklec, Y. Zhang, H.-J. Krause, H. Bousak and A.I. Braginski, Aircraft Wheel Testing with Machine-Cooled HTS SQUID Gradiometer System", IEEE Trans. Appl. Supercond. vol.9, No. 2(1999), pp. 3801.

[14]D.S. Forsyth, A. Fahr and C.E. Chapman,"An Evaluation of Artificial Neural Networks for the Classification of Eddy Current Signals", Review of Progress in QNDE, Vol 13, 879-886, 1994.

[15] M. Ramoni, P. Sebastiani, "Bayesian Methods for Intelligent Data Analysis", KMi Technical Report KMi-TR-67, July 1998 